\title{
Measuring solute concentration with terahertz time-domain spectroscopy in single and multiphase systems
}

\author{
J. Kölbel, ${ }^{\dagger} \mathbb{\Phi}$ Q. Li, ${ }^{\dagger}, \mathbb{I}$ T. Threlfall, ${ }^{\ddagger}$ and J. A. Zeitler ${ }^{*, \dagger}$ \\ $\dagger$ Department of Chemical Engineering, University of Cambridge, Cambridge, UK \\ $\ddagger$ Department of Chemistry, University of Southampton, Southampton, UK \\ ฯ Contributed equally to this work \\ E-mail: jaz22@cam.ac.uk
}

Phone: +44 (0) 1223 334783. Fax: +44 (0) 1223334796

\begin{abstract}
A versatile setup based on a microfluidics platform allows investigation of liquid samples at various temperatures with terahertz time-domain spectroscopy. The set up is applied to develop a novel method that performs temperature and concentration calibrations of liquid samples at terahertz frequencies. Other than measuring the concentration of pure liquid phase solutions, it enables the studies of local concentration of semi-crystalline systems. An equivalent solute concentration while crystallising can be calculated from the extracted absorption at low frequencies. Here, the $\mathrm{MgSO}_{4}$-water system is discussed as an example to illustrate the idea of this method.
\end{abstract}




\section{Introduction}

Terahertz time-domain spectroscopy (THz-TDS) is a useful far-infrared spectroscopy tool that is increasingly used in the field of solid state crystallography to complement x-ray diffraction methods to distinguish between polymorphs and other solid forms, as it probes collaborative motions in molecular systems that are largely influenced by the different crystalline packing. ${ }^{1}$ Its use can be extended to study crystallisation itself and it has been shown that even low amounts of crystallinity can be detected in semi-crystalline systems, ${ }^{2}$ and we recently developed a setup that allows for the in-situ investigation of crystallisation from the liquid phase over a range of concentrations and temperatures. ${ }^{3}$

Ultraviolet-visible (UV-Vis) spectroscopy has been widely applied for determining the concentration of a range of chemical species in a liquid, such as in the field of solution chemistry and water quality monitoring. ${ }^{4}$ By measuring the absorption spectrum of the sample of interest, the identity of a known compound or molecule in solution can be confirmed and using a calibration curve, the concentration of the compound can be determined quantitatively. ${ }^{5}$ However, despite its excellent sensitivity and ready availability some drawbacks of the method remain: the electronic transitions that give rise to the absorption in the UV-Vis are not very specific and often overlap and it is not always straightforward to utilise UV-Vis spectroscopy for measuring the concentration of chemicals in semi-crystalline systems, as the crystal particles will result to scattering losses and signal distortion.

In contrast, Raman spectroscopy can resolve the formation of crystals in the mid-infrared with much higher chemical specificity even in the presence of water, but it is inherently limited to study the properties of the crystals in solution rather than capturing much information about the liquid phase. ${ }^{6}$ Other optical techniques such as second harmonic generation and polarized light microscopy can both detect the onset of the crystallisation, but are not ideally suited to infer detailed mechanistic insight into the nascent molecular structures. ${ }^{7,8}$

The method that is proposed in this study is using THz-TDS to determine the concentration of the solute prior and during crystallisation. In contrast to UV-VIS spectroscopy the 
method does not require the presence of a chromophore but rather exploits the differences in relaxation dynamics of the dipole moments of the solvent molecules that strongly affect the absorption measured at terahertz frequencies. In analogy to the quantification by UV-Vis a calibration curve over a range of concentrations is required but for the THz-TDS method the sample temperature needs to be taken into account as well.

Using this method, it is possible to measure the concentration of solute in the liquid phase not just in the neat liquid but throughout phase changes such as during crystallisation. In liquid systems, the coupling to photons to the vibrational density of state (VDOS) forms the so-called microscopical peak, which is responsible for an increasing absorption coefficient with frequency observed in the terahertz region. In crystalline systems, the VDOS is depleted and instead spectral peaks are formed at discrete frequencies, thereby decreasing the baseline. Hence, terahertz spectroscopy can probe liquid relaxation dynamics as well as vibrational transitions in crystals simultaneously, represented by the baseline and the peaks on a spectrum, respectively. It is demonstrated at the example of the crystallisation of the model system magnesium sulfate heptahydrate.

\section{Experimental section}

\section{THz-TDS measurements of crystalline $\mathrm{MgSO}_{4} \cdot 7 \mathrm{H}_{2} \mathrm{O}$ in the solid state}

A sample of $\mathrm{MgSO}_{4} \cdot 7 \mathrm{H}_{2} \mathrm{O}$ (Sigma-Aldrich, Gillingham, UK) was ground using an agate mortar and a pestle, and then mixed with polyethylene $(\mathrm{PE})$ powder to $2.5 \mathrm{w} / \mathrm{w} \%$ by gentle mixing. The powder mixture was compressed into a pellet of $13 \mathrm{~mm}$ diameter using a hydraulic press (Specac Ltd., Kent, UK) at a load of 2 tons. A blank PE pellet was used as a reference. A terahertz time-domain spectrometer TeraPulse 4000 (Teraview, Cambridge, UK) was equipped with a cryostat (Janis, Massachusetts, USA) and an attached heater and controller (Lakeshore 330, Ohio, USA). The setup was capable of performing temperaturevariant transmission measurements. For each measurement, 1000 waveforms were acquired 
and averaged and a spectral resolution of $0.94 \mathrm{~cm}^{-1}$ was achieved per spectrum.

\section{$\mathrm{MgSO}_{4}$ solution measurements}

Aqueous magnesium sulfate solutions were prepared to various defined concentrations from commercial $\mathrm{MgSO}_{4} \cdot 7 \mathrm{H}_{2} \mathrm{O}$ (Sigma-Aldrich, Gillingham, UK). The experimental setup was described in detail in ${ }^{3}$ and consisted of a liquid cell inserted into a hollow metal sample holder. The temperature was controlled by a recirculating water bath. The setup could be used either under stagnant conditions, i.e. the solution was inserted at the beginning of the measurement and the liquid cell was sealed, or in the continuous flow conformation, i.e. the solution was initially kept in a heated syringe that was fixed to a syringe pump, and was slowly injected into the cell.

Once solution was injected into the liquid cell and the temperature had stabilised at the setpoint temperature (here $\sim 4^{\circ} \mathrm{C}$ ), the temperature of the cell was slowly increased at a rate of $1^{\circ} \mathrm{C} \mathrm{min}{ }^{-1}$ up to $25^{\circ} \mathrm{C}$. In the present study, calibration was therefore performed in the temperature range of $4^{\circ} \mathrm{C}$ to $25^{\circ} \mathrm{C}$ and the concentration region that was explored ranged from 0 (pure water) and $0.28 \mathrm{w} / \mathrm{w} \%\left(\mathrm{MgSO}_{4}\right.$ to water). The weight ratio/concentration is denoted as $c(\mathrm{w} / \mathrm{w} \%)$, which represents the concentration of solution.

Transmission spectra were acquired using a TeraPulse 4000 spectrometer (Teraview, Cambridge, UK) and images were acquired with an attached camera (CF-25, CrazyFire, China). Utilising the high-resolution mode of the spectrometer, time-domain waveforms of $45 \mathrm{ps}$ duration with a spectral resolution of $0.94 \mathrm{~cm}^{-1}$ were recorded. Each spectrum was calculated from the average of 15 individual waveforms, resulting in the acquisition of one spectrum per $20 \mathrm{~s}$ and covering the frequency range from $0.35 \mathrm{THz}$ to $2 \mathrm{THz}$.

Several thermocouples continuously monitored the temperature of the water bath, of the circulating water surrounding the crystallisation cell, of the syringe, and of the solution at the inlet of the cell. 


\section{Results and discussion}

\section{Crystalline $\mathrm{MgSO}_{4} \cdot 7 \mathrm{H}_{2} \mathrm{O}$}

Reference terahertz spectra of crystalline $\mathrm{MgSO}_{4} \cdot 7 \mathrm{H}_{2} \mathrm{O}$ in the solid state were acquired over a range of temperatures in order to characterise the vibrational features that are characteristic of the crystalline form. Three strong vibrational features were identified at 1.2, 1.6 and $2.8 \mathrm{THz}$ in the spectral range that was accessible using the THz-TDS setup (Figure S1). The measurement at temperatures below room temperature confirmed the presence of sharp vibrational features that underly each of the features observed at room temperature. Given that the subsequent crystallisation experiments were performed in the temperature range of $4^{\circ} \mathrm{C}$ to $25^{\circ} \mathrm{C}$, and that the presence of the liquid flow cell limits the spectral bandwidth of the spectrometer to the range of $0.35 \mathrm{THz}$ to $2 \mathrm{THz}$, the more intense feature at $1.6 \mathrm{THz}$ at $21^{\circ} \mathrm{C}$ was used to represent the behaviour of the crystalline phase (Figure 1).

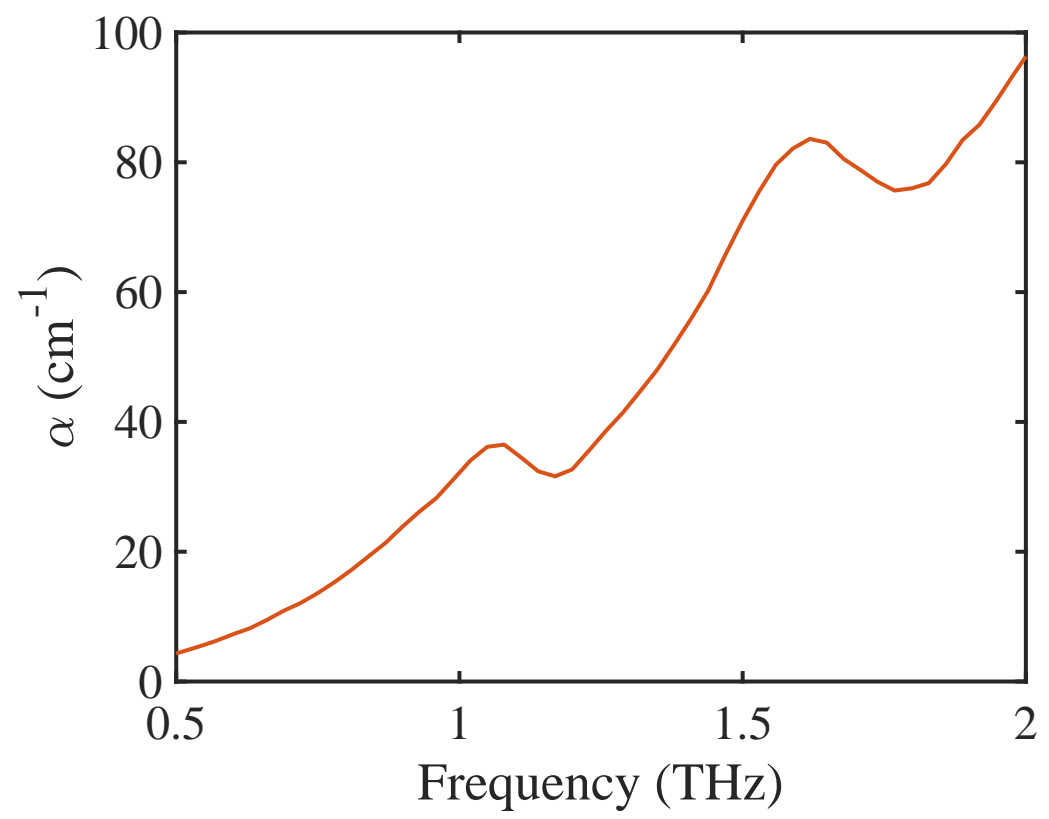

Figure 1: Solid-state terahertz spectra of crystalline $\mathrm{MgSO}_{4} \cdot 7 \mathrm{H}_{2} \mathrm{O}$ at $21^{\circ} \mathrm{C}$. 


\section{Concentration calibration}

The temperature-dependent behaviour of the liquid phase was characterised with terahertz spectroscopy for solutions over a range of concentrations $c$. For the measurement of solution at each concentration, the absorption at select frequencies was taken from the spectra and a linear fit was applied against temperature, as demonstrated in Figure 2.

$$
\alpha(c, T, \nu)=a_{1} \cdot T\left[{ }^{\circ} \mathrm{C}\right]+b_{1}
$$

where $\alpha$ is the absorption extracted from the terahertz spectra of solution at a certain concentration $c$ acquired in the temperature $(T)$ range of $4{ }^{\circ} \mathrm{C}$ to $25^{\circ} \mathrm{C}$ at a chosen frequency $\nu . \quad a_{1}$ and $b_{1}$ are the least square linear fitting parameters. Using this methodology, the parameters $a_{1}$ and $b_{1}$ for a range of concentrations are determined.

Based on the calibration methodology outlined above it is then possible to fit the change in absorption with concentration at a given temperature and frequency with $a_{2}$ and $b_{2}$ as the linear fit parameters.

$$
\alpha_{\nu, T}(c)=a_{2} \cdot c[\mathrm{w} / \mathrm{w} \%]+b_{2}
$$

Figure 3 shows the data that was derived for the case of $\mathrm{MgSO}_{4}$ solution at the three chosen frequencies: $0.5 \mathrm{THz}$ (lower bound), $1.0 \mathrm{THz}$ (high signal to noise ratio), and $1.6 \mathrm{THz}$ (centre of the crystalline peak) at a temperature of $5{ }^{\circ} \mathrm{C}$. The measurements were performed under static as well as continuous flow, and no significant deviation was identified between the different conditions, as demonstrated by the red data points in Figure 4 which correspond to the continuous flow conditions.

The calibration procedure can thus be used to extract the concentration of the solute in the liquid phase based on the measured absorption at any given temperature within calibration range and for any frequency within the spectral bandwidth of the instrument. 


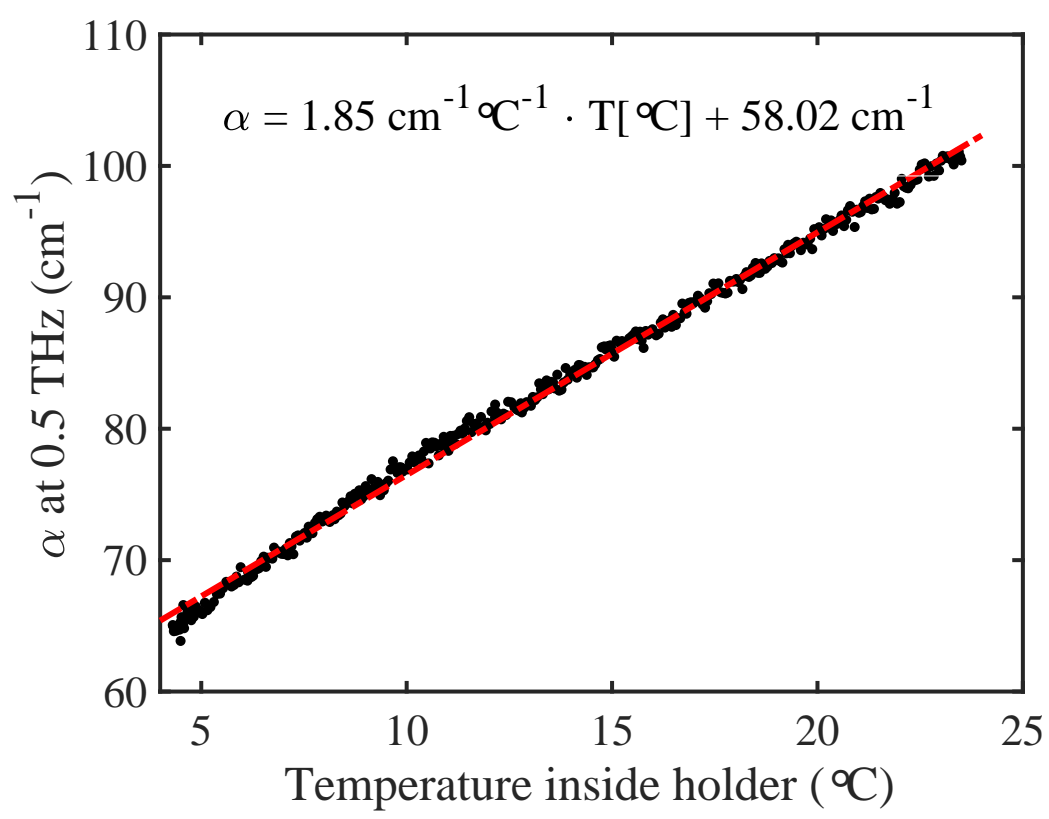

Figure 2: The linear fit of temperature $\left({ }^{\circ} \mathrm{C}\right)$ and absorption at the example of a solution of $\mathrm{MgSO}_{4}$ at a frequency of $0.5 \mathrm{THz}$ and $c=0.14 \mathrm{w} / \mathrm{w} \%$.

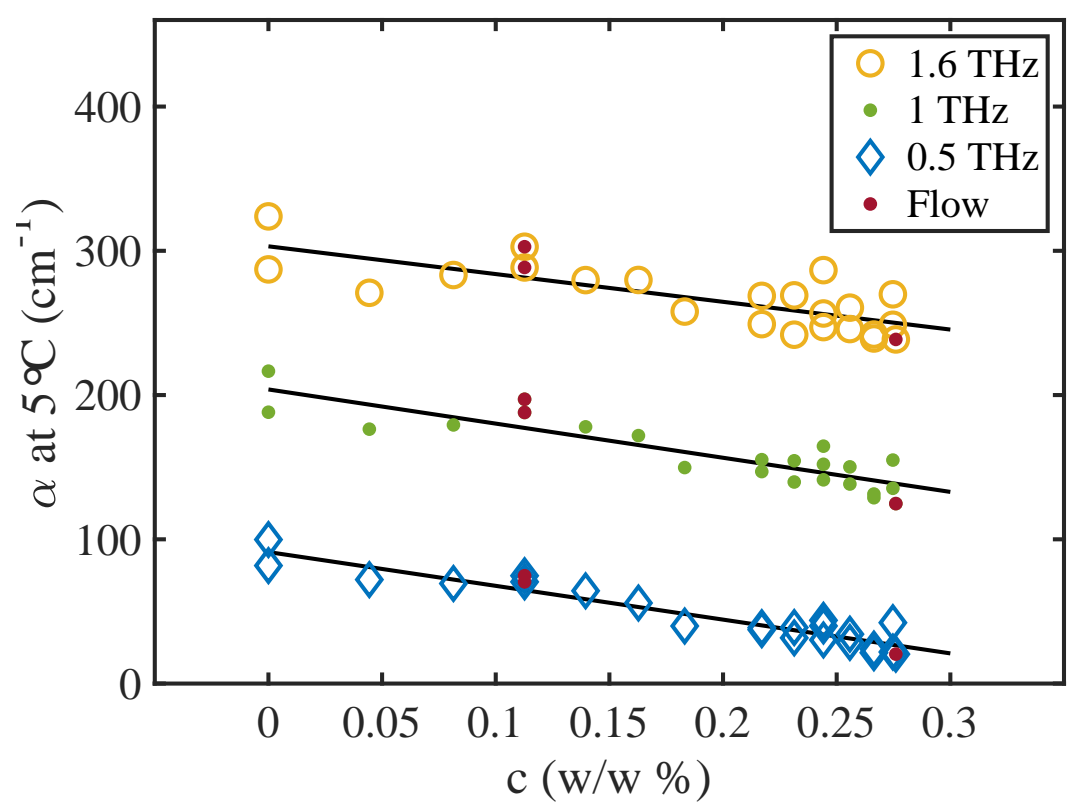

Figure 3: The calibration curve of $\mathrm{MgSO}_{4} \cdot 7 \mathrm{H}_{2} \mathrm{O}$ at $5{ }^{\circ} \mathrm{C}$. The absorption at $0.5,1.0$, and $1.6 \mathrm{THz}$ is represented by the yellow, green, and blue markers, respectively, and the red data points are the measurements using continuous flow. 


\section{Case study of $\mathrm{MgSO}_{4}$ crystallisation}

Applying the above method, it was possible to determine the local concentration of solute in the liquid phase during the crystallisation process. Compared to the case of measuring the solute concentration in the neat solution outlined above in the presence of a solid phase, i.e. the crystals, the total absorption $(\alpha)$ is the sum of the absorption from the liquid phase $\alpha_{\text {liquid }}$ and the solid phase $\alpha_{\text {solid }}$, here crystalline $\mathrm{MgSO}_{4}$ heptahydrate. To identify the local concentration in solution the contribution from $\alpha_{\text {solid }}$ can be minimised by measuring the absorption at the lower frequency end of the available bandwidth for the calibration curve. At a frequency of $0.5 \mathrm{THz}$ the crystalline phase exhibits negligible absorption (Figure 5) and $\alpha \approx \alpha_{\text {liquid. }}$. The vast majority of crystalline systems do not exhibit phonon modes at frequencies below a few hundred gigahertz and hence this method is not restricted to the $\mathrm{MgSO}_{4}$ system but generally applicable as long as the spectral characteristics have been established by a simple transmission measurement of the crystalline phase. As a general rule it can be assumed that the higher the frequency that is chosen for the analysis the more likely it would be affected by the crystalline phase when calculating the equivalent concentration.

Figure 4(a) shows the absorption at $0.5 \mathrm{THz}$ directly extracted from the terahertz spectra acquired throughout the crystallisation process, which was at the lower bound of the frequency window and no crystalline feature was expected. With the information of frequency, temperature, and absorption, the corresponding concentration of the amorphous phase was determined as shown in Figure 4(b) using the calibration curve in Figure 3. The concentration first decreased gradually due to the water depletion from the two ends of the flow cell. In the second region, the concentration increased drastically as crystals started to grow which resulted in relatively denser liquid in the area that was probed with THz-TDS. Afterwards, the concentration reduced again and slowly reached the same equilibrium point as in the beginning, which was assumed as the stable concentration at that temperature. In this way, the temperature-dependent absorption was converted to information that was independent of temperature. 

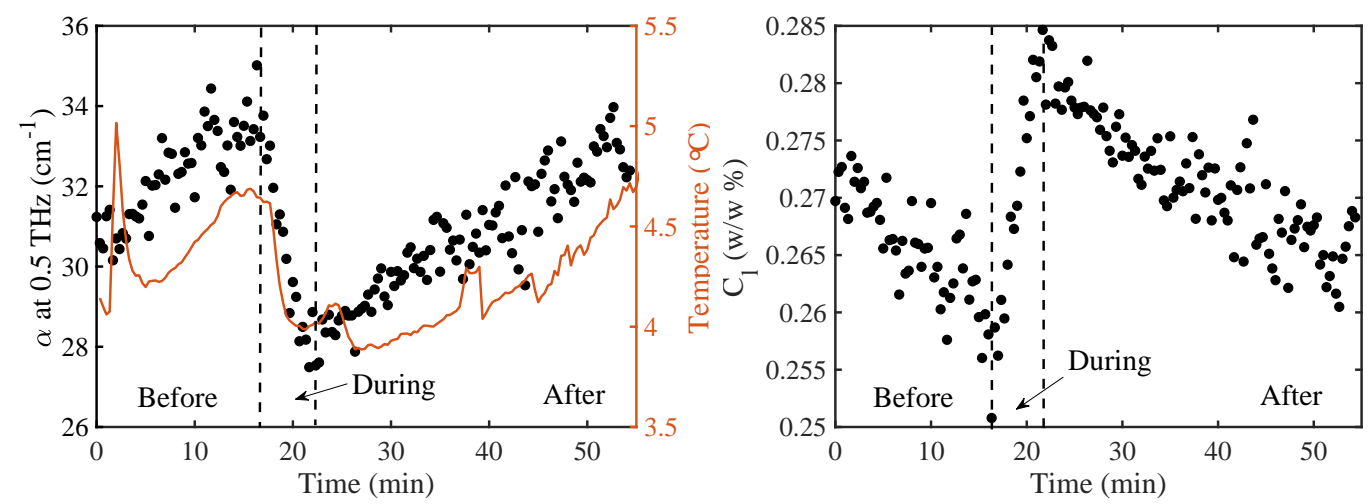

Figure 4: The absorption and calculated concentration $c_{l}$ at $0.5 \mathrm{THz}$. (a) The change of absorption over time at $0.5 \mathrm{THz}$ along with the temperature profile. (b) The calculated $c_{l}$ during the crystallisation process.

\section{Conclusions}

The newly established set up, based on microfludics and THz-TDS, has been applied to develop the proposed method of measuring solute concentration as well as the local concentration of semi-crystalline systems. By acquiring terahertz spectra of liquid samples with different concentrations at various temperatures, the calibration curve was determined by the extracted absorption at terahertz frequencies. This was then used to calculate the concentration of solutions. Furthermore, this method can also be applied to study local concentration of the liquid phase while crystallisation occurs. Using the absorption at lower frequencies is recommended in this case, since the complexity of entangled spectral features of liquid and crystalline phases at higher frequencies complicates the spectra. THz-TDS can hence be used as a complementary method for studying concentration changes in a variety of systems under different conditions.

\section{Acknowledgement}

JK thanks the EPSRC Cambridge Centre for Doctoral Training in Sensor Technologies and Applications (EP/L015889/1) and AstraZeneca for funding. QL thanks the Chinese Scholarship Council for funding. 


\section{Supporting Information Available}

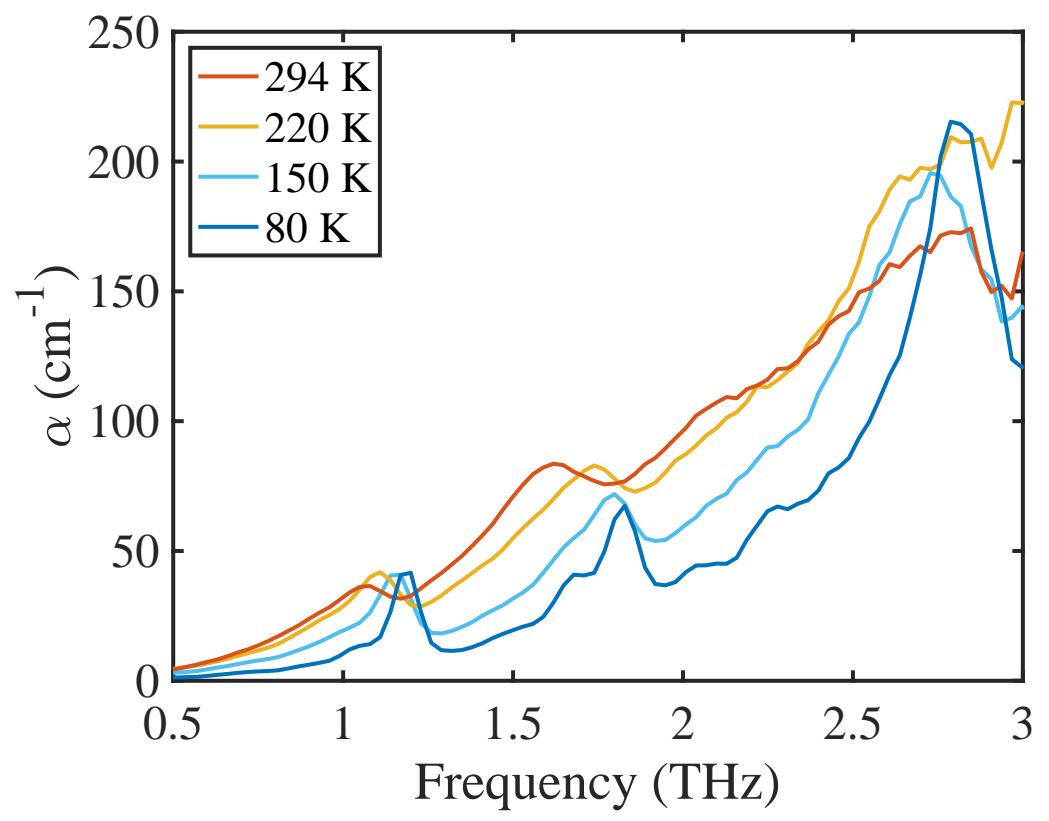

Figure 5: Solid-state terahertz spectra of crystalline $\mathrm{MgSO}_{4} \cdot 7 \mathrm{H}_{2} \mathrm{O}$ at $21^{\circ} \mathrm{C}$.

\section{References}

(1) Ruggiero, M. T.; Sibik, J.; Zeitler, J. A.; Korter, T. M. Examination of L-glutamic acid polymorphs by solid-state density functional theory and terahertz spectroscopy. The Journal of Physical Chemistry A 2016, 120, 7490-7495.

(2) Sibik, J.; Sargent, M. J.; Franklin, M.; Zeitler, J. A. Crystallization and phase changes in paracetamol from the amorphous solid to the liquid phase. Molecular pharmaceutics 2014, 11, 1326-1334.

(3) Li, Q.; Kölbel, J.; Threlfall, T.; Zeitler, J. A. Flow cell to study crystallisation processes in-situ using terahertz time-domain spectroscopy. ChemRxiv 2021, 10.33774/chemrxiv2021-mnchm. 
(4) Ojeda, C. B.; Rojas, F. S. Process analytical chemistry: applications of ultraviolet/visible spectrometry in environmental analysis: an overview. Applied Spectroscopy Reviews 2009, 44, 245-265.

(5) Brabson, G. D. Ultraviolet/Visible Absorption Spectroscopy in: ASM Handbook, Volume 10:.: Materials Characterization; ASM International, 1986.

(6) Rodríguez-Hornedo, N.; Nehm, S. J.; Seefeldt, K. F.; Pagan-Torres, Y.; Falkiewicz, C. J. Reaction crystallization of pharmaceutical molecular complexes.

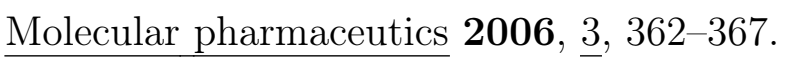

(7) Correa-Soto, C.; Trasi, N. S.; Schmitt, P. D.; Su, Y.; Liu, Z.; Miller, E.; Variankaval, N.; Marsac, P. J.; Simpson, G. J.; Taylor, L. S. Second harmonic generation microscopy as a tool for the early detection of crystallization in spray dried dispersions. Journal of pharmaceutical and biomedical analysis 2017, 146, 86-95.

(8) Basso, R. C.; Ribeiro, A. P. B.; Masuchi, M. H.; Gioielli, L. A.; Gonçalves, L. A. G.; dos Santos, A. O.; Cardoso, L. P.; Grimaldi, R. Tripalmitin and monoacylglycerols as modifiers in the crystallisation of palm oil. Food chemistry 2010, 122, 1185-1192. 


\section{TOC Graphic}

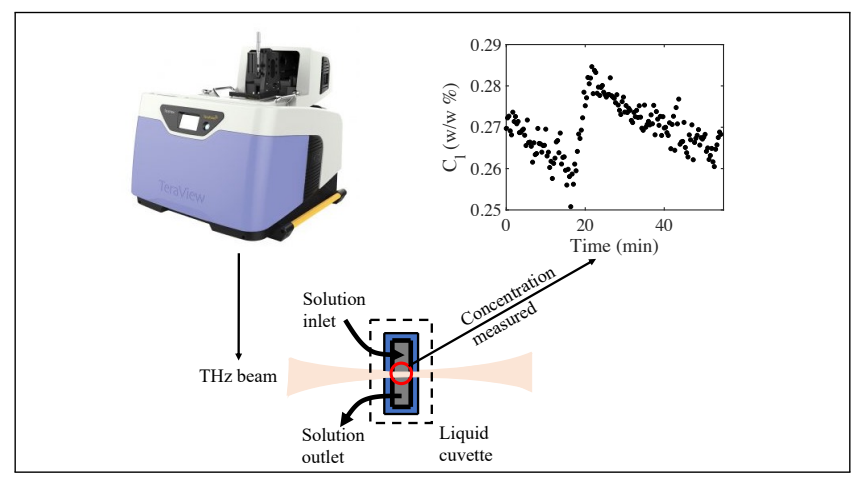

